\title{
Doubly torqued vectors and a classification of doubly twisted and Kundt spacetimes
}

\section{Carlo Alberto Mantica ${ }^{1,2} \cdot$ Luca Guido Molinari $^{2,3}$}

Received: 5 February 2021 / Accepted: 14 April 2021 / Published online: 26 April 2021

(c) The Author(s) 2021

\begin{abstract}
The simple structure of doubly torqued vectors allows for a natural characterization of doubly twisted down to warped spacetimes, as well as Kundt spacetimes down to PP waves. For the first ones the vectors are timelike, for the others they are null. We also discuss some properties, and their connection to hypersurface orthogonal conformal Killing vectors, and null Killing vectors.
\end{abstract}

Keywords Twisted spacetime $\cdot$ Kundt spacetime $\cdot$ Warped spacetime $\cdot$ Torqued vector

Mathematics Subject Classification Primary 83C20 - Secondary 53B30

\section{Contents}

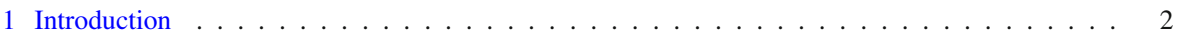

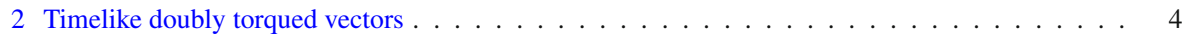

3 Timelike hypersurface orthogonal conformal Killing vectors . . . . . . . . . . . . . . . . 5

4 Null doubly torqued vectors and Kundt spacetimes . . . . . . . . . . . . . . . . . . . . . 6

5 Null hypersurface orthogonal Killing vectors . . . . . . . . . . . . . . . . . . . . 8

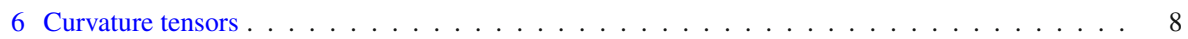

7 Conclusions ................................ 10

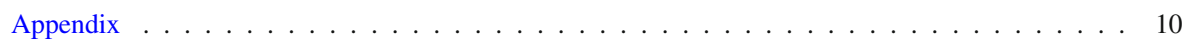

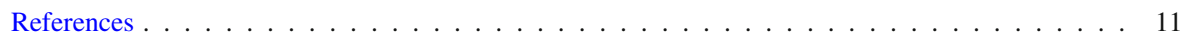

Luca Guido Molinari

luca.molinari@unimi.it

Carlo Alberto Mantica carlo.mantica@mi.infn.it

1 I.I.S. Lagrange, Via L. Modignani 65, 20161 Milan, Italy

2 I.N.F.N. sez. Milano, Via Celoria 16, 20133 Milan, Italy

3 Physics Department 'Aldo Pontremoli', Università degli Studi di Milano, Milan, Italy 


\section{Introduction}

Recently, we introduced timelike doubly torqued vectors [15]. They provide a simple characterization of $1+n$ doubly twisted spacetimes, and its subcases of twisted, doubly warped, generalized Robertson-Walker spacetimes. Remarkably, the same definition of doubly torqued vectors fits in the characterization of Kundt spacetimes: a Kundt spacetime is precisely defined by the existence of a null doubly torqued vector, and special cases as the Walker and Brinkmann metrics are naturally identified. The purpose of this paper is to present such characterizations, that are summarized in the tables of this introduction.

An important variety of spacetimes are foliations with totally umbilical spacelike Riemannian hypersurfaces of dimension $n$, parametrized by time [20]. In proper coordinates, the metric tensor has a $1+n$ block-diagonal structure. Depending on the arguments of the two scale functions $a^{2}$ and $b^{2}$, the spacetimes bear different names (Table 1).

There is a vast literature about them, since the paper by Yano [23] in 1940, who introduced doubly twisted manifolds. Warped $1+n$ spacetimes are also known as generalized Robertson-Walker $[1,6,13]$. The table includes spacetimes without name, that naturally emerge in this classification.

The same spacetimes have a tensor characterization, independent of the choice of coordinates, through the existence of a timelike-unit vector field $u_{i}$ that is vorticity-free and shear-free. Besides this description, preferred by physicists, we recently identified another one in terms of a timelike doubly torqued vector [15]:

$$
\nabla_{j} \tau_{k}=\kappa g_{j k}+\alpha_{j} \tau_{k}+\tau_{j} \beta_{k}
$$

where $\alpha_{k} \tau^{k}=0$ and $\beta_{k} \tau^{k}=0$. Despite being $u_{i}=\tau_{i} / \sqrt{-\tau^{2}}$, where $\tau^{2}=\tau_{k} \tau^{k}$, the vector $\tau_{i}$ offers a straightforward classification of the spacetimes (Table 2). In some cases, $\alpha_{i}$ and $\beta_{i}$ are gradients of scalar functions. In parallel, the vector field $u_{i}$ gets more and more specialized through requirements on the expansion parameter $\varphi$ and the acceleration $\dot{u}_{i}=u^{k} \nabla_{k} u_{i}$.

Timelike doubly-torqued vectors extend the characterizations by Bang-Yen Chen of twisted spacetimes in terms of torqued vectors $\left(\beta_{i}=0\right)$ and of warped spacetimes in terms of concircular vectors $\left(\alpha_{i}=\beta_{i}=0\right)$. They also identify other spacetimes, that do not have simple description in terms of $u_{i}$. The special case $\alpha_{i}+\beta_{i}=0$ identifies doubly torqued vectors with hypersurface orthogonal conformal Killing vectors, making contact with literature.

Surprisingly, null doubly torqued vectors exactly match the Newman-Penrose characterization of Kundt spacetimes. Since $\tau^{2}=0$ it is $\kappa=0$ in Eq. (1), and a proper rescaling gives a vector $\tau^{\prime}$ :

$$
\nabla_{i} \tau_{j}^{\prime}=\theta \tau_{i}^{\prime} \tau_{j}^{\prime}+\beta_{i}^{\prime} \tau_{j}^{\prime}+\tau_{i}^{\prime} \beta_{j}^{\prime}
$$

with $\beta^{\prime}$ the non-null component of $\beta$. Conditions on $\theta$ and $\beta^{\prime}$ give special cases, as the Walker anf Brinkmann metric of PP waves (Table 3). 
Table $1 \quad 1+$ n doubly twisted spacetimes

\begin{tabular}{ll}
\hline $1+\mathrm{n}$ spacetime & $d s^{2}=$ \\
\hline doubly twisted & $-b^{2}(t, \mathbf{q}) d t^{2}+a^{2}(t, \mathbf{q}) g_{\mu \nu}^{\star}(\mathbf{q}) d q^{\mu} d q^{\nu}$ \\
twisted & $-d t^{2}+a^{2}(t, \mathbf{q}) g_{\mu \nu}^{\star}(\mathbf{q}) d q^{\mu} d q^{\nu}$ \\
unnamed1 & $-b^{2}(t, \mathbf{q}) d t^{2}+a^{2}(t) g_{\mu \nu}^{\star}(\mathbf{q}) d q^{\mu} d q^{\nu}$ \\
unnamed2 & $-b^{2}(t, \mathbf{q}) d t^{2}+g_{\mu \nu}^{\star}(\mathbf{q}) d q^{\mu} d q^{\nu}$ \\
doubly warped & $-b^{2}(\mathbf{q}) d t^{2}+a^{2}(t) g_{\mu \nu}^{\star}(\mathbf{q}) d q^{\mu} d q^{\nu}$ \\
warped & $-d t^{2}+a^{2}(t) g_{\mu \nu}^{\star}(\mathbf{q}) d q^{\mu} d q^{\nu}$ \\
static & $-b^{2}(\mathbf{q}) d t^{2}+g_{\mu \nu}^{\star}(\mathbf{q}) d q^{\mu} d q^{\nu}$ \\
\hline
\end{tabular}

Table 2 Characterizations with timelike doubly torqued and unit vectors

\begin{tabular}{llllll}
\hline $1+n$ spacetime & $\nabla_{i} \tau_{j}=$ & & $\nabla_{i} u_{j}=$ & $\nabla_{i} \varphi=$ & \\
\hline doubly twisted & $\kappa g_{i j}+\alpha_{i} \tau_{j}+\tau_{i} \beta_{j}$ & {$[15]$} & $\varphi\left(u_{i} u_{j}+g_{i j}\right)-u_{i} \dot{u}_{j}$ & $-u_{i} \dot{\varphi}+v_{i}$ & {$[8]$} \\
twisted & $\kappa g_{i j}+\alpha_{i} \tau_{j}$ & {$[5]$} & $\varphi\left(u_{i} u_{j}+g_{i j}\right)$ & $-u_{i} \dot{\varphi}+v_{i}$ & {$[14]$} \\
unnamed1 & $\kappa g_{i j}+\tau_{i} \beta_{j}$ & & & & \\
unnamed2 & $\tau_{i} \beta_{j}$ & & & & \\
doubly warped & $\kappa g_{i j}+\tau_{i} \partial_{j} \beta$ & {$[15]$} & & & \\
warped (GRW) & $\kappa g_{i j}$ & {$[4]$} & $\varphi\left(u_{i} u_{j}+g_{i j}\right)$ & $-u_{i} \dot{\varphi}$ & [13] \\
static & $\tau_{i} \partial_{j} \beta$ & & $-u_{i} \dot{u}_{j}$ & & \\
\hline
\end{tabular}

Table 3 Kundt class spacetimes, and null doubly torqued vectors

\begin{tabular}{lc}
\hline Kundt & $d s^{2}=H(u, v, \mathbf{q}) d u^{2}-2 d u d v+$ \\
& $2 W_{\mu}(u, v, \mathbf{q}) d u d q^{\mu}+g_{\mu v}(u, \mathbf{q}) d q^{\mu} d q^{v}$ \\
& $\nabla_{i} \tau_{j}^{\prime}=\theta \tau_{i}^{\prime} \tau_{j}^{\prime}+\beta_{i}^{\prime} \tau_{j}+\tau_{i} \beta_{j}^{\prime}$ \\
\hline Unnamed 1 & $d s^{2}=H(u, v, \mathbf{q}) d u^{2}-2 d u d v+$ \\
& $2 d u d q^{\mu} \partial_{\mu}\left[\Phi_{0}(\mathbf{q})+v \Phi_{1}(\mathbf{q})\right]+$ \\
& $g_{\mu v}(u, \mathbf{q}) d q^{\mu} d q^{\nu}$ \\
& $\nabla_{i} \tau_{j}^{\prime}=\theta \tau_{i}^{\prime} \tau_{j}^{\prime}+\left(\partial_{i} \beta\right) \tau_{j}^{\prime}+\tau_{i}^{\prime}\left(\partial_{j} \beta\right)$ \\
& $d s^{2}=H(u, \mathbf{q}) d u^{2}-2 d u d v+$ \\
Unnamed 2 & $2 W_{\mu}(u, v, \mathbf{q}) d u d q^{\mu}+g_{\mu v}(u, \mathbf{q}) d q^{\mu} d q^{v}$ \\
& $\nabla_{i} \tau_{j}^{\prime}=\beta_{i}^{\prime} \tau_{j}^{\prime}+\tau_{i}^{\prime} \beta_{j}^{\prime}$ \\
Walker & $d s^{2}=H(u, v, \mathbf{q}) d u^{2}-2 d u d v+$ \\
& $2 W_{\mu}(u, \mathbf{q}) d u d q^{\mu}+g_{\mu v}(u, \mathbf{q}) d q^{\mu} d q^{v}$ \\
& $\nabla_{i} \tau_{j}^{\prime}=\theta \tau_{i}^{\prime} \tau_{j}^{\prime}$ \\
Brinkmann & $d s^{2}=H(u, \mathbf{q}) d u^{2}-2 d u d v+$ \\
& $2 W_{\mu}(u, \mathbf{q}) d u d q^{\mu}+g_{\mu v}(u, \mathbf{q}) d q^{\mu} d q^{v}$ \\
(PP waves) & $\nabla_{i} \tau_{j}^{\prime}=0$ \\
\hline
\end{tabular}




\section{Timelike doubly torqued vectors}

We obtain properties for timelike doubly torqued vectors and revisit the relations among $\tau_{i}, \kappa, \alpha_{i}, \beta_{i}$ and the scale functions $a, b>0$ of the metric, discussed in [15], to obtain new results. We refer to the coordinate frame where the space components $\tau_{\mu}$ and $u_{\mu}$ vanish, as the "comoving" frame.

Timelike doubly torqued vectors satisfy the Frobenius condition $\tau_{[i} \nabla_{j} \tau_{k]}=0$ and are hypersurface orthogonal.

This symmetry is useful:

Proposition 2.1 If $\tau_{i}$ is a timelike doubly torqued vector with $\left(\kappa, \alpha_{i}, \beta_{i}\right)$ in Eq. (1), then $\mu \tau_{i}$ is doubly torqued with $\left(\mu \kappa, \alpha_{i}+\partial_{i} \mu / \mu, \beta_{i}\right)$ provided that $\tau^{k} \partial_{k} \mu=0$.

In the comoving frame $\left(\tau_{\mu}=0\right)$ the condition means that $\partial_{t} \mu=0$.

If $\alpha_{i}=\partial_{i} \alpha$ (orthogonal to $\tau_{i}$ ), then a rescaling of $\tau_{i}$ brings it to $\alpha_{i}=0$.

Let us enquire when $\alpha_{i}$ is a gradient, i.e. is closed. Contraction of (1) with $\tau^{k}$ gives:

$$
\alpha_{j}=\nabla_{j} \log \sqrt{-\tau^{2}}-\kappa \frac{\tau_{j}}{\tau^{2}}
$$

The evaluation of $\nabla_{i} \alpha_{j}$ gives the useful identity

$$
\left(\nabla_{i} \alpha_{j}-\nabla_{j} \alpha_{i}\right) \tau^{2}=\tau_{i}\left(\nabla_{j} \kappa-\kappa \alpha_{j}-\kappa \beta_{j}\right)-\tau_{j}\left(\nabla_{i} \kappa-\kappa \alpha_{i}-\kappa \beta_{i}\right)
$$

Proposition $2.2 \alpha_{j}$ is closed if and only if $\nabla_{j} \kappa-\kappa \alpha_{j}-\kappa \beta_{j}$ is parallel to $\tau_{j}$.

In the comoving frame $\tau_{\mu}=0, \alpha_{0}=\beta_{0}=0$, with the Christoffel symbols listed in appendix, Eq. (1) for doubly torqued vectors becomes $(\mu=1, \ldots, n)$ :

$$
\begin{aligned}
& \partial_{t} \tau_{0}-\tau_{0} \partial_{t} \log b=-\kappa b^{2} \\
& \partial_{\mu} \tau_{0}-\tau_{0} \partial_{\mu} \log b=\tau_{0} \alpha_{\mu} \\
& -\partial_{\mu} \log b=\beta_{\mu} \\
& -\tau_{0} \partial_{t} \log a=\kappa b^{2}
\end{aligned}
$$

The following propositions concern the two unnamed spacetimes, respectively, and their subcases:

Proposition 2.3 In a doubly twisted spacetime, if $\alpha_{i}=0\left(\right.$ or $\alpha_{i}$ is a gradient orthogonal to $\tau$ ) then $a^{2}(t)$ only depends on time.

Proof If $\alpha_{\mu}=0$ the second equation gives $\tau_{0}(t, \mathbf{q})=F(t) b(t, \mathbf{q})$ with some function $F$. The first and last equations give $\partial_{t} \log a=\left(\partial_{t} F\right) / F(t)$.

Proposition 2.4 In a doubly twisted spacetime, $\kappa=0$ if and only if $a^{2}$ only depends on $\mathbf{q}$ (and may be included in $g_{\mu \nu}^{\star}(\mathbf{q})$ ).

Then $\alpha_{i}$ is a gradient (and can be absorbed to zero) and $\tau^{2}$ is independent of time. 
Proof The last equation gives $a^{2}$ that only depends on $\mathbf{q}$ if and only if $\kappa=0$. The first one gives $\tau_{0}=C(\mathbf{q}) b(t, \mathbf{q})$, and the second one results in $\alpha_{\mu}=\partial_{\mu} \log C(\mathbf{q})$. Then $\alpha_{i}$ is a spacetime gradient. Equation (3) gives $\alpha_{i}=\nabla_{i} \log \sqrt{-\tau^{2}}$. In the comoving frame $\alpha_{0}=0$ so that $\tau^{2}$ is independent of time.

\section{Timelike hypersurface orthogonal conformal Killing vectors}

We show that timelike doubly-torqued vectors with $\alpha_{i}+\beta_{i}=0$ coincide with hypersurface orthogonal conformal Killing vectors ( [9] Ch.11, [22] pp.69, 564). We revisit in this light some theorems, and give new ones.

Definition $3.1 \xi_{i}$ is a conformal Killing vector if $\nabla_{i} \xi_{j}+\nabla_{j} \xi_{i}=2 \kappa g_{i j}$ or, equivalently, $\nabla_{i} \xi_{j}=\kappa g_{i j}+F_{i j}$ with $F_{i j}=-F_{j i}$. It is a Killing vector if also $\kappa=0$.

Lemma 3.2 A timelike conformal Killing vector $\xi_{i}$ is hypersurface orthogonal if and only if: $F_{j k}=\alpha_{j} \xi_{k}-\xi_{j} \alpha_{k}, \alpha_{k} \xi^{k}=0$, i.e.

$$
\nabla_{i} \xi_{j}=\kappa g_{i j}+\alpha_{j} \xi_{k}-\xi_{j} \alpha_{k}
$$

Proof By the Frobenius theorem, a vector is hypersurface orthogonal if and only if $0=\xi_{[i} \nabla_{j} \xi_{k]}=\xi_{i}\left(\nabla_{j} \xi_{k}-\nabla_{k} \xi_{j}\right)+$ cyclic permutations i.e. $\xi_{i} F_{j k}+\xi_{j} F_{k i}+\xi_{k} F_{i j}=0$. A contraction with $\xi^{i}$ gives $\xi^{2} F_{j k}+\xi_{j}\left(F_{k i} \xi^{i}\right)-\xi_{k}\left(F_{j i} \xi^{i}\right)=0$. It is always possible to choose $\alpha_{k} \xi^{k}=0$, as $\alpha_{k}-\alpha_{j} \xi_{k} \xi^{j} / \xi^{2}$ does the job.

Proposition 3.3 Doubly torqued vectors with $\alpha_{i}=-\beta_{i}$ are hypersurface orthogonal conformal Killing vectors. They are hypersurface orthogonal Killing vectors if also $\kappa=0$.

In Ref. [15] we showed that a doubly twisted spacetime is doubly warped if and only if $\alpha_{i}=\partial_{i} \alpha$ and $\beta_{i}=\partial_{i} \beta$ in (1) (see Table 2). Since they are both orthogonal to $\tau$ we may rescale $\tau$ such that $\alpha_{i}=-\partial_{i} \beta$ and obtain $\nabla_{i} \tau_{j}=\kappa g_{i j}-\left(\partial_{i} \beta\right) \tau_{j}+\tau_{i}\left(\partial_{j} \beta\right)$, a conformal Killing vector. Therefore:

Proposition 3.4 A spacetime is doubly warped if and only if it is equipped with a hypersurface orthogonal conformal Killing vector with closed vector $\alpha_{i}$.

With $\alpha_{i}=-\beta_{i}$ in (4), we read that $\alpha_{j}$ is closed if and only if $\nabla_{j} \kappa$ is proportional to $\tau_{k}$. Therefore, we have the statement (Theorem 1 in [21]): A spacetime is doubly warped if and only if it is equipped with a hypersurface orthogonal conformal Killing vector with $\partial_{i} \kappa$ parallel to $\xi_{i}$.

Moreover, if $\tau$ is closed $\left(\alpha_{i}=\beta_{i}\right)$ then $\alpha_{i}=\beta_{i}=0$ : the spacetime is generalized Robertson-Walker (Cor. 2 in [21]).

A doubly torqued vector with $\kappa=0, \alpha_{i}=-\beta_{i}$ is a hypersurface orthogonal Killing vector. Since $\alpha_{i}$ and $-\alpha_{i}$ are gradients (Prop.2.4), the spacetime is doubly warped. Then $a^{2}$ is a function of $t$ and $b^{2}$ is a function of $\mathbf{q} . \kappa=0$ means that $\partial_{t} a=0$ i.e. $a$ is a constant. The metric $d s^{2}=-b^{2}(\mathbf{q}) d t^{2}+a^{2} g_{\mu \nu}^{\star}(\mathbf{q}) d q^{\mu} d q^{v}$ has the form of a static spacetime [22] p.283. 


\section{Null doubly torqued vectors and Kundt spacetimes}

A Kundt spacetime is defined by the presence of a null geodesic congruence that is expansion-free, shear-free, and twist-free [22] Ch.31, [3,11,17,18]. We show that it precisely means that it admits a doubly torqued null vector field.

We begin with some facts on null doubly torqued vectors.

The contraction of $\nabla_{i} \tau_{j}=\kappa g_{i j}+\alpha_{i} \tau_{j}+\tau_{i} \beta_{j}$ with $\tau^{j}$ gives $\kappa=0$. Then:

$$
\nabla_{i} \tau_{j}=\alpha_{i} \tau_{j}+\tau_{i} \beta_{j}, \quad \alpha_{k} \tau^{k}=0, \beta_{k} \tau^{k}=0
$$

Contraction with $\tau^{i}$ gives that $\tau$ is geodesic: $\tau^{i} \nabla_{i} \tau_{j}=0$.

For null vectors one considers the optical scalars [19]:

$$
\Theta=\frac{1}{d-2} \nabla_{k} \tau^{k}, \quad \omega^{2}=-\nabla_{[k} \tau_{j]} \nabla^{k} \tau^{j}, \quad \sigma^{2}=\nabla_{(k} \tau_{j)} \nabla^{k} \tau^{j}-(d-2) \Theta^{2}
$$

where $d$ is the dimension of spacetime. It is simple to prove that all the three optical scalars vanish for null doubly torqued vectors. In particular, the vanishing of the twist $\left(\omega^{2}=0\right)$ is the condition for $\tau$ to be hypersurface orthogonal.

Since $\tau^{2}=0, \alpha_{i}=a \tau_{i}+\alpha_{i}^{\prime}$ where $\alpha^{\prime}$ is a spacelike vector orthogonal to $\tau$, and $\beta=b \tau_{i}+\beta_{i}^{\prime}$. Then, for a null doubly torqued vector, with $\theta=a+b$, it is

$$
\nabla_{i} \tau_{j}=\theta \tau_{i} \tau_{j}+\alpha_{i}^{\prime} \tau_{j}+\tau_{i} \beta_{j}^{\prime}
$$

We now turn to Kundt spacetimes and show that (8) is precisely the equation for the congruence. Let $\ell_{i}$ be the geodesic null congruence, and $n_{i}$ a second null vector field with $n_{i} \ell^{i}=-1 . \hat{h}_{i j}=g_{i j}+\ell_{i} n_{j}+n_{i} \ell_{j}$ is the projection on the space orthogonal to $\ell$ and $n$. Consider the decomposition

$$
\begin{aligned}
\nabla_{i} \ell_{j} & =\left(\hat{h}_{i}^{l}-\ell_{i} n^{l}-n_{i} \ell^{l}\right)\left(\hat{h}_{j}^{m}-\ell_{j} n^{m}-n_{j} \ell^{m}\right) \nabla_{l} \ell_{m} \\
& =\left(\hat{h}_{i}^{l}-\ell_{i} n^{l}\right)\left(\hat{h}_{j}^{m}-\ell_{j} n^{m}\right) \nabla_{l} \ell_{m} \\
& =\hat{h}_{i}^{l} \hat{h}_{j}^{m} \nabla_{l} \ell_{m}+\ell_{i} \ell_{j}\left(n^{l} n^{m} \nabla_{l} \ell_{m}\right)-\hat{h}_{i}^{l} \ell_{k} n^{m} \nabla_{l} \ell_{m}-\ell_{i} n^{l} \hat{h}_{j}^{m} \nabla_{l} \ell_{m}
\end{aligned}
$$

The omitted terms contain $\ell^{l} \nabla_{l} \ell_{m}=0$ (the field is geodesic) and $\ell^{m} \nabla_{l} \ell_{m}=0$. The first term is the projection onto the subspace of dimension $d-2$ orthogonal to $\ell_{i}$ and $n_{i}$, and is decomposed into expansion, shear and twist:

$$
\hat{h}_{i}^{l} \hat{h}_{j}^{m} \nabla_{l} \ell_{m}=\frac{\nabla_{l} \ell^{l}}{d-2} \hat{h}_{i j}+\hat{\sigma}_{i j}+\hat{\omega}_{i j}
$$

For Kundt spacetimes these terms are zero, and we have the known statement (we shift to the letter $\tau_{i}$ ):

$$
\nabla_{i} \tau_{j}=\left(n^{l} n^{m} \nabla_{l} \tau_{m}\right) \tau_{i} \tau_{j}-\left(\hat{h}_{i}^{l} n^{m} \nabla_{l} \tau_{m}\right) \tau_{j}-\tau_{i}\left(\hat{h}_{i}^{m} n^{l} \nabla_{l} \tau_{m}\right)
$$


Theorem 4.1 A spacetime is Kundt if and only if there is a doubly torqued null vector field, Eq. (6) or (8).

The property $\lambda \tau_{i}=\nabla_{i} f$ (hypersurface orthogonality) offers a rescaling of $\tau$ that makes it a closed vector:

Proposition 4.2 The vector $\tau_{i}^{\prime}=\lambda \tau_{i}$ is null doubly torqued, closed, and

$$
\nabla_{i} \tau_{i}^{\prime}=\theta \tau_{i}^{\prime} \tau_{j}^{\prime}+\beta_{i}^{\prime} \tau_{j}^{\prime}+\tau_{i}^{\prime} \beta_{j}^{\prime}
$$

where the vector $\beta^{\prime}$ is the component of $\beta$ not aligned with $\tau$.

Proof The evaluation gives: $\nabla_{i} \tau_{j}^{\prime}=\left(\alpha_{i}+\partial_{i} \lambda / \lambda\right) \tau_{j}^{\prime}+\tau_{i}^{\prime} \beta_{j}$. Since $\tau_{i}^{\prime}$ is closed, it is $\left(\alpha_{i}-\beta_{i}+\partial_{i} \lambda / \lambda\right) \tau_{j}^{\prime}=\left(\alpha_{j}-\beta_{j}+\partial_{j} \lambda / \lambda\right) \tau_{i}^{\prime}$. Then: $\alpha_{i}+\partial_{i} \lambda / \lambda=\beta_{i}+\gamma \tau_{i}^{\prime}$ and $\nabla_{i} \tau_{j}^{\prime}=\gamma \tau_{i}^{\prime} \tau_{j}^{\prime}+\beta_{i} \tau_{j}^{\prime}+\tau_{i}^{\prime} \beta_{j}$. Next, being $\beta_{i} \tau^{i}=0$ and $\tau$ null, it is $\beta=b \tau_{i}+\beta_{i}^{\prime}$. The expression is obtained.

The metric of a Kundt spacetime in coordinates adapted to the null vectors is:

$$
d s^{2}=H(u, v, \mathbf{q}) d u^{2}-2 d u d v+2 W_{\mu}(u, v, \mathbf{q}) d u d q^{\mu}+g_{\mu v}(u, \mathbf{q}) d q^{\mu} d q^{v}
$$

The coordinates $u$ and $v$ refer to the subspace spanned by $\tau_{i}$ and $n_{i}$, where $\tau_{u}=-1$, $\tau_{v}=0, \tau_{\mu}=0, \alpha_{u}^{\prime}=\beta_{u}^{\prime}=0$. Equation (8) gives the following relations:

$$
\theta=\frac{1}{2} \frac{\partial H}{\partial v}, \quad \alpha_{v}^{\prime}=\beta_{v}^{\prime}=0, \quad \alpha_{\mu}^{\prime}=\beta_{\mu}^{\prime}=-\frac{1}{2} \frac{\partial W_{\mu}}{\partial v}
$$

It turns out that the metric is evaluated with the vector (9).

We have three special cases:

(i) $\partial H / \partial v=0$ corresponds to $\theta=0$

(ii) $\partial W_{\mu} / \partial v=0$, i.e. $\alpha_{i}^{\prime}=\beta_{i}^{\prime}=0$. It is $\nabla_{i} \tau_{j}=\theta \tau_{i} \tau_{j}$. This recurrent case gives the Walker metric [12].

(iii) $\partial H / \partial v=0$ and $\partial W_{\mu} / \partial v=0$ equivalent to $\theta=0, \alpha_{i}^{\prime}=\beta_{i}^{\prime}=0$. This case gives the Brinkmann metric (PP wave, i.e. plane-fronted waves with parallel propagation) $[2,17]$.

Another special case is $\beta^{\prime}$ closed. The equation $\nabla_{i} \beta_{j}^{\prime}=\nabla_{j} \beta_{i}^{\prime}$ gives: 1) $\partial_{\mu} \beta_{v}^{\prime}=$ $\partial_{\nu} \beta_{\mu}^{\prime}$ i.e. $W_{\mu}=\partial_{\mu} \Phi(u, v, \mathbf{q})$ for some potential; 2$) \partial_{u} \beta_{\mu}^{\prime}=0$, then $\Phi$ does not depend on $u$; 3) $\partial_{v} \beta_{\mu}^{\prime}=0$, then $\Phi$ is a linear function of $v$. In summary: $\beta^{\prime}$ closed implies $W_{\mu}(v, \mathbf{q})=\partial_{\mu} \Phi_{0}(\mathbf{q})+v \partial_{\mu} \Phi_{1}(\mathbf{q})$, (in Table 3).

This case is realized in the solutions of the Einstein-Maxwell equations in vacuo, or with electromagnetic field aligned to $\tau\left(F_{i j} \tau^{j} \propto \tau_{i}\right)$, or with the cosmological constant. For this problem $H$ is a quadratic function of $v$ (Eqs. 77 and 112 in [18]). 


\section{Null hypersurface orthogonal Killing vectors}

In analogy with timelike vectors, we consider null doubly torqued vectors with $\alpha_{i}=$ $-\beta_{i}$. They coincide with (hypersurface orthogonal) null Killing vectors, and describe a subclass of Kundt spacetimes [7].

Proposition 5.1 A null hypersurface orthogonal Killing vector is a doubly torqued vector with $\alpha_{i}=-\beta_{i}$.

A null doubly torqued vector $\nabla_{i} \tau_{j}=\alpha_{i} \tau_{j}-\tau_{i} \alpha_{j}$ is a Killing vector.

Proof The hypothesis are: $\nabla_{i} \tau_{j}=F_{i j}\left(F_{i j}=-F_{j i}\right)$ and $\tau_{i}=\lambda \nabla_{i} f$. Then: $F_{i j}=$ $\left(\nabla_{i} \lambda\right) \nabla_{j} f+\lambda \nabla_{i} \nabla_{j} f$. Subtraction of $F_{j i}$ gives $F_{i j}=\frac{1}{2} \frac{\nabla_{i} \lambda}{\lambda} \tau_{j}-\frac{1}{2} \frac{\nabla_{j} \lambda}{\lambda} \tau_{i}$. Since $F_{i j} \tau^{j}=$ 0 , the vector $\tau$ is doubly torqued with $\alpha_{i}=-\beta_{i}$.

A doubly torqued vector is hypersurface orthogonal and, if $\beta_{i}=-\alpha_{i}$ it is $\nabla_{i} \tau_{j}+$ $\nabla_{j} \tau_{i}=0$ i.e. $\nabla_{i} \tau_{j}=F_{i j}=-F_{j i}$.

The metric in $d=4$ is given in [22] p.380. If $\tau_{i}$ is also closed, then $\nabla_{i} \tau_{j}=0$ and PP waves are obtained.

\section{Curvature tensors}

The integrability conditions for a null or timelike doubly torqued vector are:

$$
\begin{aligned}
R_{j k l m} \tau^{m}= & g_{k l}\left(\nabla_{j} \kappa-\kappa \alpha_{j}\right)-g_{j l}\left(\nabla_{k} \kappa-\kappa \alpha_{k}\right)+\left(\nabla_{j} \alpha_{k}-\nabla_{k} \alpha_{j}\right) \tau_{l} \\
& +\tau_{k}\left(\nabla_{j} \beta_{l}-\beta_{j} \beta_{l}\right)-\tau_{j}\left(\nabla_{k} \beta_{l}-\beta_{k} \beta_{l}\right)
\end{aligned}
$$

The contraction of the Ricci tensor with $\tau^{m}$ is obtained:

$$
R_{k m} \tau^{m}=-(n-1) \nabla_{k} \kappa+\kappa\left(n \alpha_{k}+\beta_{k}\right)+\tau^{j} \nabla_{j} \alpha_{k}+\tau_{k}\left(\alpha^{j} \beta_{j}+\nabla_{j} \beta^{j}\right)
$$

Then, a null $\tau$ is eigenvector if and only if $\tau^{j} \nabla_{j} \alpha_{k} \propto \tau_{k}$.

Lemma 6.1 For null doubly torqued vectors:

$$
\begin{aligned}
& \tau_{i} \nabla_{j}\left(\alpha_{k}-\beta_{k}\right)+\tau_{j} \nabla_{k}\left(\alpha_{i}-\beta_{i}\right)+\tau_{k} \nabla_{i}\left(\alpha_{j}-\beta_{j}\right)=0 \\
& \tau^{k} \nabla_{k}\left(\alpha_{i}-\beta_{i}\right)=\tau_{i}\left(\alpha^{k} \beta_{k}-\beta^{2}\right)
\end{aligned}
$$

Proof The first Bianchi identity $R_{j k l m}+R_{k l j m}+R_{l j k m}=0$ is contracted with $\tau^{m}$ and the expressions (12) are inserted, with $\kappa=0$.

Contraction with $\tau^{k}$ gives the other identity.

The property of Weyl or Riemann compatibility for vectors and symmetric tensors is presented in [16]. Riemann compatibility implies Weyl compatibility.

Theorem 6.2 A timelike doubly torqued vector is Weyl compatible:

$$
\tau_{i} C_{j k l m} \tau^{m}+\tau_{j} C_{k i l m} \tau^{m}+\tau_{k} C_{i j l m} \tau^{m}=0
$$


A null doubly torqued vector with $\alpha_{i}$ closed or with $\beta_{i}=C \alpha_{i}$ with $C \neq 1$ a constant, is Riemann compatible

$$
\tau_{i} R_{j k l m} \tau^{m}+\tau_{j} R_{k i l m} \tau^{m}+\tau_{k} R_{i j l m} \tau^{m}=0
$$

and is an eigenvector of the Ricci tensor.

Proof Multiplication of (12) by $\tau_{i}$ and a cyclic sum give:

$$
\begin{aligned}
& \tau_{i} R_{j k l m} \tau^{m}+\tau_{j} R_{k i l m} \tau^{m}+\tau_{k} R_{i j l m} \tau^{m} \\
& =\left[\tau_{i}\left(\nabla_{j} \alpha_{k}-\nabla_{k} \alpha_{j}\right)+\tau_{j}\left(\nabla_{k} \alpha_{i}-\nabla_{i} \alpha_{k}\right)+\tau_{k}\left(\nabla_{i} \alpha_{j}-\nabla_{j} \alpha_{i}\right)\right] \tau_{l} \\
& \quad-g_{i l}\left[\kappa\left(\tau_{j} \alpha_{k}-\tau_{k} \alpha_{j}\right)-\left(\tau_{j} \nabla_{k} \kappa-\tau_{k} \nabla_{k} \kappa\right)\right]+ \\
& \quad-g_{j l}\left[\kappa\left(\tau_{k} \alpha_{i}-\tau_{i} \alpha_{k}\right)-\left(\tau_{k} \nabla_{i} \kappa-\tau_{i} \nabla_{k} \kappa\right)\right]+ \\
& \quad-g_{k l}\left[\kappa\left(\tau_{i} \alpha_{j}-\tau_{j} \alpha_{i}\right)-\left(\tau_{i} \nabla_{j} \kappa-\tau_{j} \nabla_{i} \kappa\right]\right.
\end{aligned}
$$

If $\tau_{i}$ is null it is $\kappa=0$. If also $\nabla_{j} \alpha_{k}=\nabla_{k} \alpha_{j}$ or if $\beta_{i}=C \alpha_{i}$ then the cyclic sum is zero (in the second case, use the Lemma).

The contraction of (15) with $g^{j l}$ gives $\tau_{i} R_{k m} \tau^{m}=\tau_{k} R_{i m} \tau^{m}$. Then $\tau$ is an eigenvector of the Ricci tensor.

Let $\tau_{i}$ by timelike. The contraction of the Weyl tensor with $\tau$ is:

$$
\begin{aligned}
C_{j k l m} \tau^{m}= & R_{j k l m} \tau^{m}+\frac{1}{n-2}\left[\tau_{j} R_{k l}-\tau_{k} R_{j l}\right] \\
& +\frac{1}{n-2} g_{k l}\left[R_{j m} \tau^{m}-\frac{R \tau_{j}}{n-1}\right]-\frac{1}{n-2} g_{j l}\left[R_{k m} \tau^{m}-\frac{R \tau_{k}}{n-1}\right]
\end{aligned}
$$

Multiplication by $\tau_{i}$ and a ciclic sum give:

$$
\begin{aligned}
& \tau_{i} C_{j k l m} \tau^{m}+\tau_{j} C_{k i l m} \tau^{m}+\tau_{k} C_{i j l m} \tau^{m} \\
& =\left[\tau_{i}\left(\nabla_{j} \alpha_{k}-\nabla_{k} \alpha_{j}\right)+\tau_{j}\left(\nabla_{k} \alpha_{i}-\nabla_{i} \alpha_{k}\right)+\tau_{k}\left(\nabla_{i} \alpha_{j}-\nabla_{j} \alpha_{i}\right)\right] \tau_{l} \\
& \quad+\frac{1}{n-2} g_{k l}\left\{\left(\tau_{i} R_{j m}-\tau_{j} R_{i m}\right) \tau^{m}-(n-2)\left[\kappa\left(\tau_{i} \alpha_{j}-\tau_{j} \alpha_{i}\right)-\left(\tau_{i} \nabla_{j} \kappa-\tau_{j} \nabla_{i} \kappa\right]\right\}\right. \\
& \quad+\frac{1}{n-2} g_{j l}\left\{\left(\tau_{k} R_{i m}-\tau_{i} R_{k m}\right) \tau^{m}-(n-2)\left[\kappa\left(\tau_{k} \alpha_{i}-\tau_{i} \alpha_{k}\right)-\left(\tau_{k} \nabla_{i} \kappa-\tau_{i} \nabla_{k} \kappa\right)\right]\right\} \\
& \quad+\frac{1}{n-2} g_{i l}\left\{\left(\tau_{j} R_{k m}-\tau_{k} R_{j m}\right) \tau^{m}-(n-2)\left[\kappa\left(\tau_{j} \alpha_{k}-\tau_{k} \alpha_{j}\right)-\left(\tau_{j} \nabla_{k} \kappa-\tau_{k} \nabla_{k} \kappa\right)\right]\right\}
\end{aligned}
$$

The contraction of the Ricci tensor with $\tau$ is (13). The cyclic sum for the Weyl tensor simplifies:

$$
\begin{aligned}
& \tau_{i} C_{j k l m} \tau^{m}+\tau_{j} C_{k i l m} \tau^{m}+\tau_{k} C_{i j l m} \tau^{m} \\
&= {\left[\tau_{i}\left(\nabla_{j} \alpha_{k}-\nabla_{k} \alpha_{j}\right)+\tau_{j}\left(\nabla_{k} \alpha_{i}-\nabla_{i} \alpha_{k}\right)+\tau_{k}\left(\nabla_{i} \alpha_{j}-\nabla_{j} \alpha_{i}\right)\right] \tau_{l} } \\
&+\frac{1}{n-2} g_{k l}\left[\tau_{i}\left(-\nabla_{j} \kappa+\kappa\left(2 \alpha_{j}+\beta_{j}\right)+\tau^{m} \nabla_{m} \alpha_{j}\right)-\tau_{j}\left(-\nabla_{i} \kappa+\kappa\left(2 \alpha_{i}+\beta_{i}\right)+\tau^{m} \nabla_{m} \alpha_{i}\right)\right] \\
&+\frac{1}{n-2} g_{j l}\left[\tau_{k}\left(-\nabla_{i} \kappa+\kappa\left(2 \alpha_{i}+\beta_{i}\right)+\tau^{m} \nabla_{m} \alpha_{i}\right)-\tau_{i}\left(-\nabla_{k} \kappa+\kappa\left(2 \alpha_{k}+\beta_{k}\right)+\tau^{m} \nabla_{m} \alpha_{k}\right)\right] \\
&+\frac{1}{n-2} g_{i l}\left[\tau_{j}\left(-\nabla_{k} \kappa+\kappa\left(2 \alpha_{k}+\beta_{k}\right)+\tau^{m} \nabla_{m} \alpha_{k}\right)-\tau_{k}\left(-\nabla_{j} \kappa+\kappa\left(2 \alpha_{j}+\beta_{j}\right)+\tau^{m} \nabla_{m} \alpha_{j}\right)\right]
\end{aligned}
$$


For timelike vectors, contraction of (12) by $\tau^{l} \tau^{k}$ gives:

$$
\begin{aligned}
0 & =\tau^{2}\left(\nabla_{j} \kappa-\kappa \alpha_{j}\right)-\tau_{j} \tau^{k} \nabla_{k} \kappa+\tau^{2} \tau^{k}\left(\nabla_{j} \alpha_{k}-\nabla_{k} \alpha_{j}\right)+\tau^{2} \tau^{l} \nabla_{j} \beta_{l}-\tau_{j} \tau^{l} \tau^{k} \nabla_{k} \beta_{l} \\
& =\tau^{2}\left[\nabla_{j} \kappa-\kappa\left(2 \alpha_{j}+\beta_{j}\right)-\tau^{k} \nabla_{k} \alpha_{j}\right]-\tau_{j}\left(\tau^{k} \nabla_{k} \kappa+\tau^{2} \alpha^{k} \beta_{k}\right)
\end{aligned}
$$

With this identity and (4) the cyclic sum is zero.

Some remarks:

- For a timelike doubly torqued vector: $C_{j k l m} \alpha^{j} \beta^{k} \tau^{m}=0$.

- Weyl compatibility (14) guarantees that all doubly twisted spacetimes are purely electric [10].

- Null hypersurface orthogonal Killing vectors are Riemann compatible.

- A Kundt spacetime with Weyl compatible vector $\tau$ is type II(d) in the Bel-Debever classification (Table 4 in [17]).

\section{Conclusions}

We showed that the structure of doubly torqued vector is the necessary and sufficient condition for the spacetime to be doubly twisted (timelike vector) or a Kundt spacetime (null vector). A simple classification of relevant subcases follows, with connection to other characterizations in terms of Killing or conformal Killing vectors.

\section{Appendix}

The Christoffel symbols for the doubly-twisted metric:

$$
\begin{aligned}
& \Gamma_{0,0}^{0}=\frac{\partial_{t} b}{b}, \quad \Gamma_{\mu, 0}^{0}=\frac{b_{\mu}}{b}, \quad \Gamma_{0,0}^{\mu}=\frac{b b^{\mu}}{a^{2}}, \quad \Gamma_{\mu, 0}^{\rho}=\frac{\partial_{t} a}{a} \delta_{\mu}^{\rho}, \quad \Gamma_{\mu, \nu}^{0}=\frac{a \partial_{t} a}{b^{2}} g_{\mu \nu}^{*}, \\
& \Gamma_{\mu, v}^{\rho}=\Gamma_{\mu, \nu}^{* \rho}+\frac{a_{v}}{a} \delta_{\mu}^{\rho}+\frac{a_{\mu}}{a} \delta_{\nu}^{\rho}-\frac{a^{\rho}}{a} g_{\mu \nu}^{*}
\end{aligned}
$$

where $a_{\mu}=\partial_{\mu} a$ and $a^{\mu}=g^{* \mu v} a_{\nu}$, and the same is for $b$.

The Christoffel symbols for the Kundt metric (that are needed in this paper. Taken from [18]) :

$$
\Gamma_{u, u}^{u}=\frac{1}{2} \frac{\partial H}{\partial v}, \quad \Gamma_{\mu, u}^{u}=\frac{1}{2} \frac{\partial W_{\mu}}{\partial v} \quad \Gamma_{u, v}^{u}=\Gamma_{v, v}^{u}=\Gamma_{\mu, v}^{u}=\Gamma_{\mu, v}^{u}=0
$$

Funding Open access funding provided by Università degli Studi di Milano within the CRUI-CARE Agreement.

Open Access This article is licensed under a Creative Commons Attribution 4.0 International License, which permits use, sharing, adaptation, distribution and reproduction in any medium or format, as long as you give appropriate credit to the original author(s) and the source, provide a link to the Creative Commons licence, 
and indicate if changes were made. The images or other third party material in this article are included in the article's Creative Commons licence, unless indicated otherwise in a credit line to the material. If material is not included in the article's Creative Commons licence and your intended use is not permitted by statutory regulation or exceeds the permitted use, you will need to obtain permission directly from the copyright holder. To view a copy of this licence, visit http://creativecommons.org/licenses/by/4.0/.

\section{References}

1. Alías, L., Romero, A., Sánchez, M.: Uniqueness of complete spacelike hypersurfaces of constant mean curvature in generalized Robertson-Walker spacetimes. Gen. Relativ. Gravit. 27(1), 71-84 (1995)

2. Coley, A., Milson, R., Pelavas, N., Pravda, V., Pravdová, A., Zalaletdinov, R.: Generalizations of PP-wave spacetimes in higher dimensions. Phys. Rev. D 67(10), 104020 (2003)

3. Coley, A., Hervik, S., Papadopoulos, G., Pelavas, N.: Kundt spacetimes. Class. Quantum Grav. 26(10), 105016 (2009)

4. Chen, B.-Y.: A simple characterization of generalized Robertson-Walker space-times. Gen. Relativ. Gravit. 46, 1833 (2014)

5. Chen, B.-Y.: Rectifying submanifolds of Riemannian manifolds and torqued vector fields. Kragujevac J. Math.s 41(1), 93-103 (2017)

6. Chen, B.-Y.: Differential Geometry of Warped Product Manifolds and Submanifolds. World Scientific, Singapore (2017)

7. Dautcourt, G.: Vacuum gravitational fields with a null Killing vector. Gen. Relativ. Gravit. 52, 10 (2020)

8. Ferrando, J.J., Morales, J.A., Portilla, M.: Inhomogeneons space-times admitting isotropic radiation: vorticity-free case. Phys. Rev. D 46(2), 578-584 (1992)

9. Hall, G.S.: Symmetries and Curvature Structure in general Relativity. World Scientific, Singapore (2004)

10. Hervik, S., Ortaggio, M., Wylleman, L.: Minimal tensors and purely electric or magnetic spacetimes of arbitrary dimension. Class. Quantum Grav. 30, 165014 (2013)

11. Kundt, W.: The plane-fronted gravitational waves. Z. Physik 163, 77-86 (1961)

12. Leistner, T.: Screen bundles of Lorentzian manifolds and some generalizations of pp-waves. J. Geom. Phys. 56(10), 2117-2134 (2006)

13. Mantica, C.A., Molinari, L.G.: Generalized Robertson-Walker space-times: a survey. Int. J. Geom. Methods Mod. Phys. 14(3), 1730001 (2017)

14. Mantica, C.A., Molinari, L.G.: Twisted Lorentzian manifolds: a characterization with torse-forming time-like unit vectors. Gen. Relativ. Gravit. 49, 51 (2017)

15. Mantica, C.A., Molinari, L.G.: A simple characterization of doubly twisted spacetimes. Int. J. Geom. Methds Mod. Phys. (2021). https://doi.org/10.1142/S0219887821500791

16. Mantica, C.A., Molinari, L.G.: The Jordan algebras of Riemann, Weyl and curvature compatible tensors. Colloq. Math. arXiv:1910.03929 [math.DG] (2019). https://doi.org/10.4064/cm8067-10-2020

17. Ortaggio, M., Pravda, V., Pravdová, A.: Algebraic classification of higher dimensional spacetimes based on null alignment. Class. Quantum Grav. 30, 013001 (2013)

18. Podolský, J., Žofka, M.: General Kundt spacetimes in higher dimensions. Class. Quantum Grav. 26, 105008 (2009)

19. Poisson, E.: An Advanced Course in General Relativity, University of Guelph (2002)

20. Ponge, R., Reckziegel, H.: Twisted product in pseudo-Riemannian geometry. Geom. Dedicata 48, 15-25 (1993)

21. Ramos, M.P.M., Vaz, E.G.L.R., Carot, J.: Double warped space-times. J. Math. Phys. 44(10), 4839 (2003)

22. Stephani, H., Kramer, D., MacCallum, M., Hoenselaers, C., Herlt, E.: Exact Solutions of Einstein's Field Equations, 2nd edn. Cambridge University Press, Cambridge (2003)

23. Yano, K.: Conformally separable quadratic differential forms. Proc. Imp. Acad. 16(3), 83-86 (1940)

Publisher's Note Springer Nature remains neutral with regard to jurisdictional claims in published maps and institutional affiliations. 